\title{
The role of immunohistochemistry expression of COX-2 in differentiating pigmented benign and malignant skin neoplasms
}

\author{
Maryam Ghasemi ${ }^{1}$, Parvaneh Afshar ${ }^{2}$, Somayeh Sheidaei ${ }^{1}$, Yosef Moeini ${ }^{1}$, Lale Vahedi Larijani*1, 3
}

Received: 15 Feb 2018

Published: 25 Jul 2019

\begin{abstract}
Background: Skin cancer is one of the most common types of cancer and its annual mortality rate is increasing. The induction enzyme of cyclooxygenase COX-2 causes biosynthesis of prostaglandin and thromboxane during inflammation of the body. Increasing the expression of COX-2 has an important role in the development and progression of malignant epithelial cancers and other types of cancers. Considering the diagnostic status of the marker, this study aimed to evaluate the expression of COX-2 for diagnosis and differentiation of benign skin pigmented neoplastic lesions from malignant melanoma types.

Methods: In this diagnostic study, the immunohistochemistry of COX-2 maker in 82 paraffin blocks of pigmented benign and malignant skin neoplasms of patients (49 men; 33 women) and its association with clinicopathological features of the tumor was evaluated. Data were analyzed using chi-squared and t test in SPSS18. Significance level was set at less than 5\%.

Results: The findings showed that 20 patients $(24.3 \%)$ had malignant melanoma and 13 had significant COX-2 (3+ High), while COX-2 marker was not detected in other benign and malignant pigmented skin neoplasms $(\mathrm{p}<0.001)$. A significant association was found between COX-2 marker and grade $(\mathrm{p}<0.001)$, but there was no significant correlation with other clinicopathological tumor criteria. Sensitivity, specificity, PPV and NPV value of the COX-2 marker were $65 \%, 100 \%, 89.9 \%$, and $100 \%$, respectively.

Conclusion: Because of the high level of COX-2 in malignant melanoma skin marker, it can be used to distinguish benign and malignant neoplastic lesions (SCC and BCC) from melanoma and to provide effective therapeutic strategies through specific COX-2 enzyme inhibitors.
\end{abstract}

Keywords: Cyclooxygenase-2, Neoplastic lesions, Skin cancer, Melanoma, Immunohistochemistry

Conflicts of Interest: None declared

Funding: Mazandaran University of Medical Sciences

*This work has been published under CC BY-NC-SA 1.0 license.

Copyright $₫$ Iran University of Medical Sciences

Cite this article as: Ghasemi M, Afshar P, Sheidaei S, Moeini Y, Vahedi Larijani L. The role of immunohistochemistry expression of COX-2 in differentiating pigmented benign and malignant skin neoplasms. Med J Islam Repub Iran. 2019 (25 Jul);33:75. https://doi.org/10.47176/mjiri.33.75

\section{Introduction}

Skin is the largest organ of the body that acts as the first physical defensive barrier to innate immunity throughout the life of each person while continuously expanding (1, 2). It is important for the body to prevent or take care of any serious skin lesion and damage. Due to the complex structure of skin tissue, there is the possibility of a wide range of diseases, including tumors in the epidermis, epi-

Corresponding author: Dr Lale Vahedi Larijani, Ialevahedi@gmail.com

1. Department of Pathology, School of Medicine, Mazandaran University of Medica Sciences, Sari, Iran

2. Research and Development Unit of Referral Laboratory, Deputy of Health Management, Mazandaran University of Medical Sciences, Sari, Iran

3. Gastrointestinal Cancer Research Center, Mazandaran University of Medical Sciences, Sari, Iran dermal appendages, and dermal tissue $(2,3)$.

Pigmentation disorders form a wide range of common skin and mucosal diseases. Studies in many countries have shown a high prevalence of skin cancer, which is caused by the abnormal growth of cells with the ability to spread to other parts of the body (4). The disease is seen more frequently in people with light skin, hair and eyes $(5,6)$,

$\uparrow$ What is "already known" in this topic:

Skin cancer is one of the most common types of cancer, and its annual mortality rate is increasing.

$\rightarrow$ What this article adds:

Because there is a high level of COX-2 marker in malignant melanoma skin, it can be used to distinguish benign and malignant neoplastic lesions ( $\mathrm{SCC}$ and $\mathrm{BCC}$ ) from melanoma and to provide effective therapeutic strategies through specific COX-2 enzyme inhibitors. 
and aged 14-35 years compared to Asian and African races $(4,7)$. Epidemiological studies in the United States, England, and Australia have indicated that skin cancers are the most commonly reported human tumors $(8,9)$, accounting for approximately $25 \%-40 \%$ of all types of cancers worldwide $(2,10-13)$. Skin cancer is highly prevalent in the Middle Eastern countries (14, 15). Limited studies registered in Iran have reported about 10-15 new cases of skin cancer per 100000 populations annually, ranking skin cancer as the most common cancer nationwide (16-18). This cancer is also very common in Mazandaran province and is ranked third among all types of common cancers (16).

The melanocytic tumors are classified into benign and malignant forms $(8,19)$. The malignant form of skin cancer is divided into 2 categories: (1) nonmelanoma skin cancers (NMSCs), which include basal cell carcinoma (BCCs) and squamous cell carcinoma (SCC); and (2) noncommon skin cancers $(\sim 1 \%)$ and malignant melanoma (MM) $(20,21)$. Typically, the prevalence of NMSCs is 18 to 20 times more than that of melanoma $(20,22)$, and about $80 \%$ of them are BCCs, and the remaining $20 \%$ are SCC $(4,23)$. However, it is estimated that about $30 \%-50 \%$ of BCC cases and $30 \%$ of SCC are not included in the cancer registration systems (4).

Due to the slow growth of $\mathrm{BCC}$, the risk of damaging the surrounding tissues and spreading to distant parts is unlikely, while destruction, metastasis, and dissemination are commonly observed in all types of SCC and MM (24).

BCC and SCC are rarely fatal $(6,25)$. Although less than $1 \%$ of skin cancer cases belong to melanoma, the melanoma form is responsible for about $75 \%$ of skin cancer deaths, about 1 person per hour $(6,26)$. According to the WHO reports, annually, about 65000 deaths (less than $0.1 \%$ of cancer deaths) occur due to this particular type of cancer in the United states $(25,27)$. In 2012, about 55000 of the 232000 cases of the melanoma reported throughout the world led to death (25). The highest rates of the melanoma have been reported in Australia and New Zealand (6). According to WHO estimates, the Middle East countries might be faced with $80 \%-100 \%$ increase in the cancer death rates over the next decade $(28,29)$.

Several genetic changes are associated with the pathogenesis of SCC, with the most important causes being cell cycle impairment, apoptosis, DNA repair, cell differentiation, expression of cyclooxygenase type 2 (COX-2), and telomerase activity (24).

Given the high prevalence of skin cancer, which has increased more than 2 times over the past 3 decades, a definite and rapid diagnosis of the disease, especially in the MM form, can prevent the progressive course of the disease by correct decision making about the primary effective measures and complementary therapies $(4,6,26)$.

Histopathological examinations have been recognized as valuable tools for skin cancer diagnosis. However, some forms of BCC and SCC are viewed as pigmented lesions and several cases of pigmented skin neoplasms are found as borderline. Thus, there are limitations in the definitive diagnosis of other pigmented lesions and in distinguishing them from one another (e.g. distinguishing SCC from Pseudoepitheliomatous hyperplasia or Keratoacanthoma) $(2,30,31)$.

To distinguish benign and malignant neoplasms, it is important to determine the prognosis, predict survival, design the therapeutic protocol, and identify many tumor markers in the specific staining techniques using immunohistochemistry (30).

Several tumor markers have been investigated and the results of which are different or even contradictory. The cyclooxygenase (COXs) enzymes are protein tumor markers that play a facilitating role in converting arachidonic acid to prostaglandin. Different genes encode COX-1 and COX-2 isozymes. One of the most important marker tumors is COX-2. An inducible enzyme plays the role of prostaglandin and thromboxane production during inflammation.

Many studies have shown increased expression of COX-2 enzyme in transformed cells in various types of human neoplasms (32-35), which can also be used as an important indicator in diagnosing the pigmented skin cancer (36-38). The use of selective COX-2 inhibitors and nonsteroidal anti-inflammatory drugs (NSAIDs) has also led to a relative improvement in the therapeutic trend of advanced cancers (38).

There are limited and different studies on the role of COX-2 in the pathogenesis of melanoma. Some reports suggested that the COX-2 overexpression in malignant melanomas may be associated with the development of this disorder. Other studies have found an increase in COX-2 protein instead of primary melanoma cells in inflammatory or metastatic cells (39). Previous studies on human and mouse melanoma cells showed that the COX-2 induces prostaglandin E2 production and malignant melanoma through its effect on the immune system, as the COX-2 inhibitors should be able to play a therapeutic role in this cancer (40).

Limited studies have been conducted in different countries, especially in Iran, on the relationship between the incidence of COX-2 and the clinicopathological parameters of pigmented cancers and its value in distinguishing benign and malignant pigmented neoplasms. Thus, the present study was conducted to detect and distinguish benign and malignant neoplastic lesions by COX-2 protein marker using immunohistochemistry as a prime step for other applied studies to investigate the relationship between the incidence of this tumor marker with prognosis, survival, and targeted therapy with specific drugs.

\section{Methods}

\section{Sample selection}

This descriptive analytical study was conducted on 82 skin tissue blocks in patients with pigmented skin cancer "based on coding ICD-O-3 provided by the WHO" available in the archives of the pathology laboratory of Bu-Ali Sina hospital, affiliated to Mazandaran University of Medical Sciences (Sari, Iran), during 2015-2014. The nontumoral adjacent tissue of the paraffin embedded nevi samples was used as negative control and paraffin embedded tumor tissue of samples diagnosis of colorectal adenocarcinoma as positive control. Two pathologists separately 
confirmed all the diagnostic steps of the samples. After rediagnosis of malignancy, the COX-2 immunohistochemical (IHC) staining intensity was evaluated for all samples.

In the first step, in addition to examining the available data in the above period, all skin pigmented specimens were diagnosed with malignant and benign neoplasms and the data were extracted from the medical records of the patients. After taking the required paraffinated blocks from the archives, slides were prepared and stained using Hematoxylin-Eosin staining method. During the examination of the slides, in addition to rediagnosing the presence or absence of malignancy, other microscopic examination parameters, such as lymphatic invasion, vascular invasion, and tumor differentiation, were also evaluated and recorded.

\section{Evaluation of COX-2 marker}

For the COX-2 IHC staining intensity test, an appropriate paraffinated block was selected from each sample (which had sufficient tumor tissue without necrosis and bleeding) and 3 micron sections were prepared using a microtome. After deparaffinization and rehydration, the samples were washed with phosphate buffered saline (PBS) and then exposed to $3 \%$ hydrogen peroxide for 20 30 minutes to inhibit the endogenous (tissue) peroxidase activity. Subsequently, after rewashing with PBS buffer, the antigen retrieval step was performed by autoclaving the samples in $0.01 \mathrm{M}$ Tris buffer with $\mathrm{pH}=9$ for 20 minutes at $120^{\circ} \mathrm{C}$. Protein block serum-free solution (code $\mathrm{X} 0909$, DAKO) was also used for 10 minutes to reduce the binding of nonspecific antibodies.

The specimens (dilution of 1/100) were then incubated for an hour with Monoclonal Mouse Anti-Human COX-2 antibody, Clone CX-294, Dako, Denmark (anti-COX-2). After rinsing with PBS buffer, the samples were treated with HRP-conjugated secondary antibody (Code-No.
K4061 empfohlen, Dako EnVision) for 30 minutes to detect the immune response.

After rinsing with PBS buffer, the samples were subjected to diaminobenzidine substrate for 10 minutes. Background stain was performed by Hematoxylin. In each series, all steps were also performed for positive and negative controls.

The cell staining was due to the cytoplasmic COX-2 marker; if stained positive, the cytoplasm's color would change to brown. The final report was based on optical microscope observations (400X) and the intensity of COX-2 staining of the cells as a semi-quantitative and estimated way within 4 grades, negative $(-)$, poor $(+)$, moderate $(+2)$, and strong $(+3)($ Table 1$)$. The grades less than +3 was considered as low COX -2 staining and grades +3 as high COX-2 staining (41).

\section{Statistical analysis}

Data were analyzed using SPSS Statistics V18, and significance level was set at $\mathrm{p}<0.05$. Mean and standard deviation were applied to describe quantitative data (age) and frequency distribution for qualitative data (sex) in each group. Student $t$ test was used for comparing patients' age, chi-squared test for gender comparisons, and logistic regression for examining the difference in incidence rate of COX-2. Sensitivity, specificity, positive predictive values (PPV), negative predictive values (NPV), and their verification ratio were determined to evaluate the diagnostic power of this marker.

\section{Results}

In total, 82 samples of $49(60 \%)$ males and $33(40 \%)$ females, with the mean \pm SD age of $50.2 \pm 3.83$-years were evaluated in this study. There was no statistically significant difference between the mean age and sex. Table 2 shows the incidence of COX-2 marker and its staining

Table 1. The protocol of the severity and colour intensity reporting in the cytoplasmic background cells of COX-2 expression IHC method (a semi quantitative and estimated assessment).

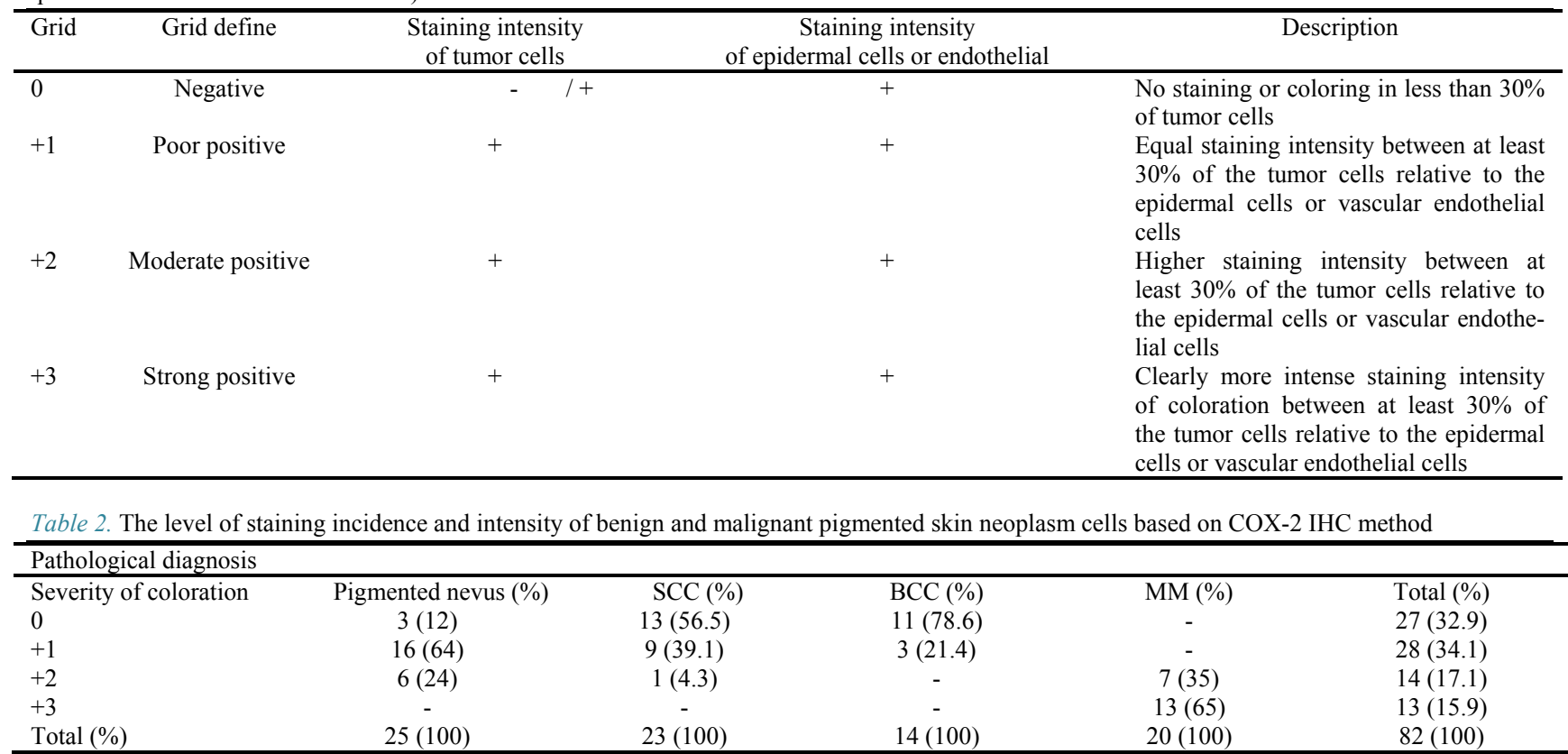


intensity in the case and control groups.

According to the pathological assessments of the COX2 IHC staining intensity test, $13(65 \%)$ out of 20 samples of MM group had a high grade $(+3)$ and the rest of $35 \%$ had a moderate $(+2)$ grade. In other groups, no +3 staining was observed (Figs. 1 and 2). There was a significant correlation between incidence of COX-2 marker with pathologic diagnosis and histological grade of benign and malignant pigmented neoplasms $(\mathrm{p}<0.05)$.

Since the negative control group was selected from the healthy tissue adjacent to each tumor, both case and control groups were fully matched for age and sex. There was a significant difference between the above group and the malignant melanoma group and other benign and malignant pigmented neoplasms in the evaluation of results $(\mathrm{p}<0.001)$.

\section{Discussion}

Skin diseases, particularly skin tumors, are major health concerns. These diseases are considered important in diagnosis, treatment, high costs of treatment, follow-up, pressure, and causing personal and social problems. Considering the high incidence of skin cancer in the world, including Iran, rapid, definite, reliable, and early diagnosis of the disease and identifying new and applied therapeutic methods to control and eliminate the disease can be an effective approach to ensure the health of the individuals and society.

The present findings indicated that the prevalence of SCC (28\%) was higher than BCC (17.1\%) and MM $(24.4 \%)$. There was no significant relationship between these malignancies and sex. The mean age of the disease was 50.2 years, which was lower than other studies in Mashhad, Gilan, Yazd that reported the mean age of over 60 years.

Although the results of other studies indicated a high prevalence of skin cancer in the elderly, often in the sixth decade of life and subsequent years, skin cancer in children and adolescents and those younger than 35 years are due to the lack of skin care against UV (A, B, C), especially in the hot months of the year (with an average radiation index of about 9). Also, the disease in the middle-
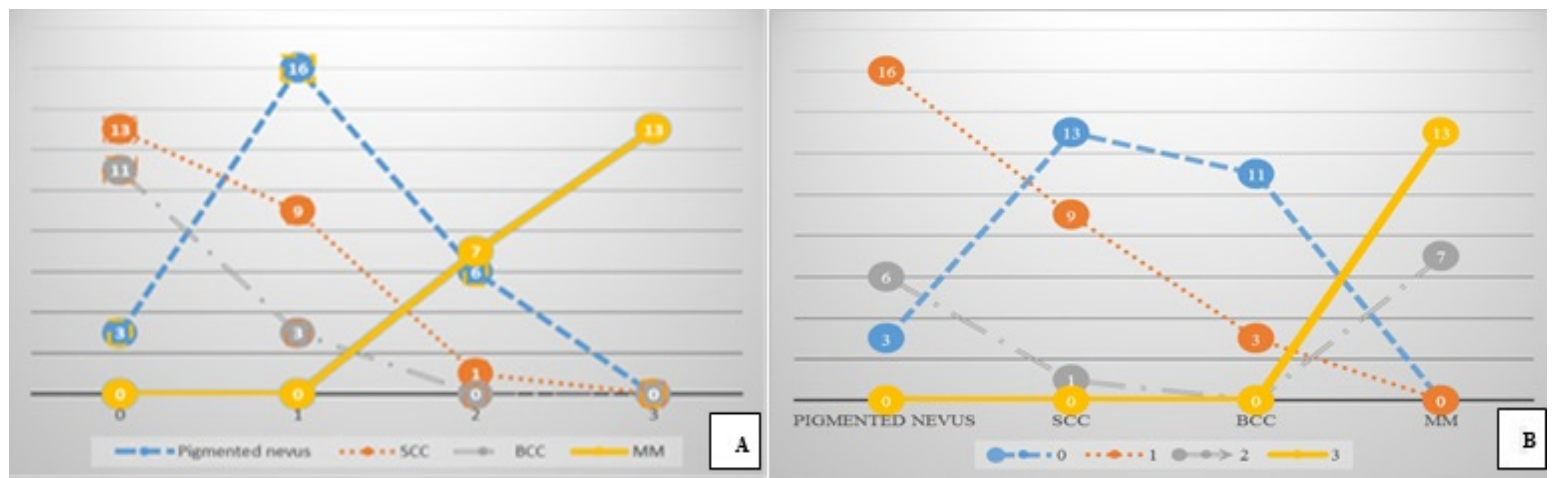

Fig. 1. Comparing the incidence and intensity expression of COX-2 in pigmented benign and malignant skin neoplasms ( $\mathrm{n}=82)$ based on; A: Severity of correlation B: Pathological diagnosis
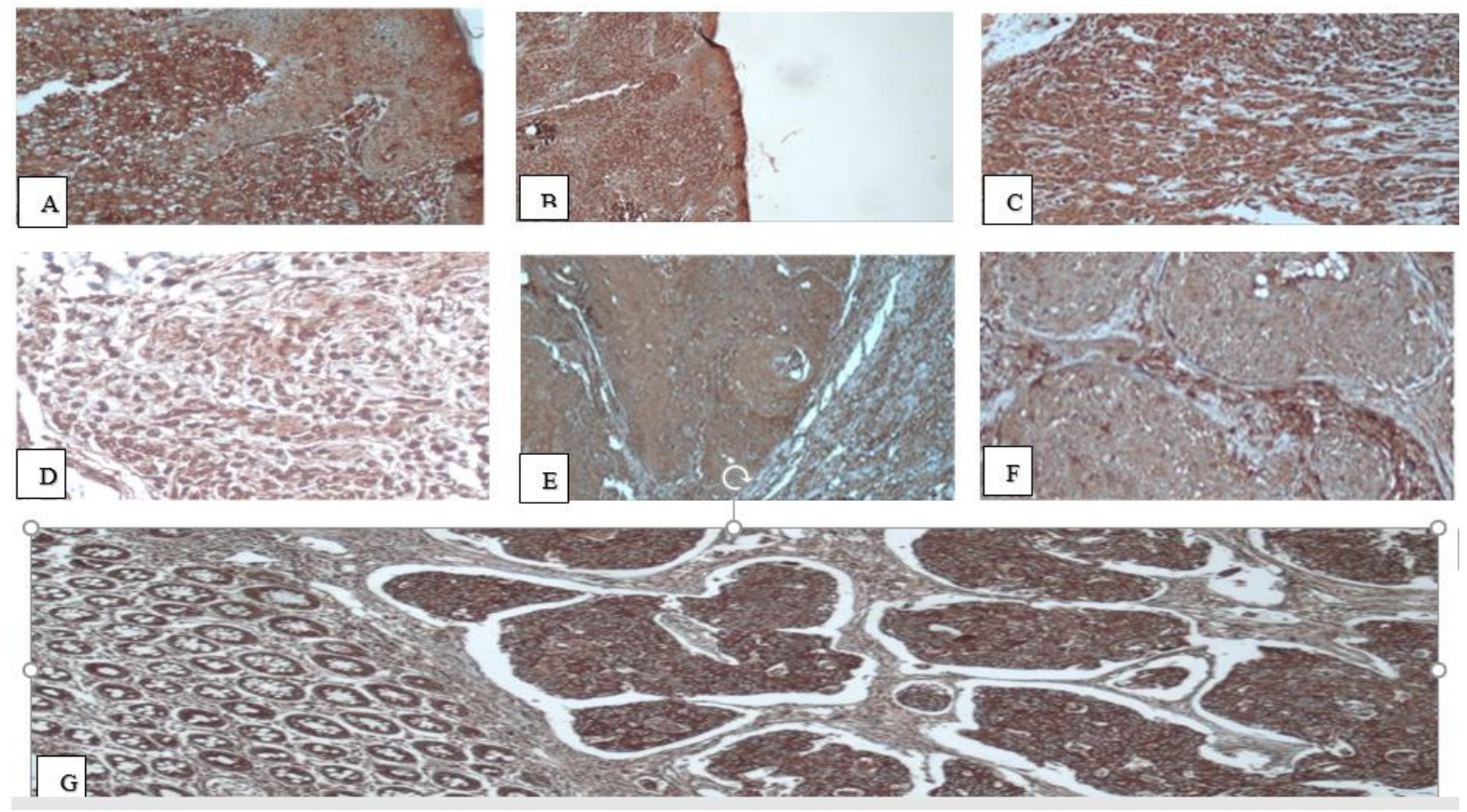

Fig. 2. The level of cytoplasmic staining intensity of benign and malignant pigmented skin neoplasm cells based on COX-2 IHC method; Positive staining MM cell (+3) “A: X400, B: X100, C: X40” Negative staining "D: Pigmented naïve.X400, E: BCC. X100, F: SCC. X100" Positive control "G: Colorectal adenocarcinoma X 400". 
aged and the elderly occur due to a weakened immune system $(4,42)$.

The UV radiation from the energy saving light bulb and welding with metal electrodes used in electrical arc technique are of the other environmental factors causing this complication (14). Given that limited studies have been performed on this subject in Iran, it is impossible to easily identify the norms and conditions of this disease. The UV radiation in people with light or dark skin causes the immune system to be inhibited by altering Langerhans cells (antigen-presenting immune cells) of the skin and mucosa, increasing the expression of immunosuppressive neuropeptides, releasing the cytokines, melanocins and inflammatory mediators from keratinocytes, melanocytes, and neurites (24). The UV radiation, after oxidative stress, increases the formation of free radicals of oxygen (active and unstable compounds with high affinity to react, invade, and damage to other molecules, including DNA or cell genome, proteins, and carbohydrates). The ROS molecules cause loss of membrane integrity and its transformation into malignant melanocytes with a cytotoxic and genotoxic role by initially stimulating the melanosomes within the melanocyte $(24,43)$.

Since the distinction between primary melanoma cells and other benign skin diseases is not possible simply by histopathologic methods and there is even the possibility of reporting the errors, detecting primary melanoma is considered as one of the important challenges of laboratory diagnosis. Studies have shown the role of the COX-2 marker expression in the ability to distinguish between nevi and types of primary and secondary melanocytes (44) Goulet et al found a very low COX-2 expression level in mild and primary melanoma but a significantly high COX-2 expression level in all inflammatory epithelial cells and metastatic melanoma. A similar study in the Netherlands showed a significant relationship between the mean COX-2 expression in mild and primary melanocytes (metastatic). Kubus et al examined 75 normal nevi, 43 atypical nevi (from children), and 15 malignant melanomas (from adults) using 2 methods of routine hematoxylin-eosin staining and the COX-2 IHC staining method. The results indicated that although all melanocytic nevi and melanomas showed a reaction to COX-2, significant differences were observed only between benign and malignant melanocytic tumors, but this difference was not statistically significant in melanocytic nevi. Therefore, the ability of the COX-2 marker to detect nevi and melanoma was successful (19), which is consistent with the findings of the present study.

To investigate the relationship between the histological grade of the disease and the incidence rate of COX-2 markers in the melanoma and the ability to distinguish the melanoma from the benign nevi, Goulet et al (17), in 2003, studied 46 specimens, including dysplastic nevi, stage I melanoma, stage II melanoma, stage III melanoma, stage IV melanoma, melanoma with metastasis to the lymph nodes, and melanoma with metastasis to the rest of the body. Their results showed the COX-2 enzyme in all keratinocytes, cutaneous fibroblasts, inflammatory cells adjacent benign nevi, and primary dermal melanomas, but not in any of the benign cells and primary dermal melanoma. The level of COX-2 protein expression was highly significant in all 13 metastatic melanoma samples prepared from the lymph nodes beneath the epithelium or other parts of the body and the inflammatory cells around them. These data showed that the COX-2 levels not only play a key role in metastatic melanoma, but also may be effective in treating these diseases with COX-2 inhibitors (39). In a similar study by Zelenay et al on 101 cases of malignant melanoma and 28 associated metastases, a high and severe level of COX-2 expression was detectable in $95 \%$ of all melanomas. In addition, its level remained constant in a variety of dependent metastases. They observed a significant correlation between the COX-2 IHC staining intensity and the thickness of the tumor and found a significant correlation between the COX-2 IHC staining intensity and the disease persistence by the Kaplan-Meier curve (40).

Another important finding of the present study was the significant relationship between higher grades and the severity of the disease invasion. Few studies have been conducted on this subject. The results of a semiquantitative study by Denkert et al on 28 cases of primary dermal melanoma and 4 benign nevi showed that 26 $(93 \%)$ of melanomas were positive for COX-2 markers and $68(68 \%)$ had medium to severe grades. In the study of de Souza do Nascimento et al, in Brazil, on 49 oral melanocytic lesions (39 intramucosal nevi, 4 dermal nevi, 13 oral primary melanomas, and 8 skin melanomas), the COX-2 incidence rate was strong in all 13 oral primary melanomas, but negative in intramucosal and dermal nevi (45). Chwirot et al examined 33 cases of primary melanoma (Clark's level I/II) and 58 benign nevi and determined a high level of COX-2 expression in melanomas, with a sensitivity of $91 \%$ and a specificity of $51.7 \%$. They reported this enzyme as the first immunohistochemical marker to distinguish the primary melanomas from the benign melanocytic lesions. In this survey, sensitivity, specificity, PPV, and NPV of the marker were $65 \%$, $100 \%, 89.9 \%$, and $100 \%$, respectively, indicating a significant relationship between histological grade and severity of COX-2 marker in the melanoma and the ability to distinguish the melanoma from benign dermal nevi.

Early and proper diagnosis of the diseases is one of the most important therapeutic approaches for adopting effective strategies to significantly reduce the progress and the severity of the disease as the priorities of the health policy of Iran. Considering the increased incidence rate of skin cancer in the country and the changed pattern (46), rapid and early diagnosis of precancerous lesions and prevention of developing the malignant melanoma are important health care issues. The direct and strong association between the incidence of COX-2 markers and the pathologic diagnosis of the malignant melanoma can help distinguish malignant and benign pigmented skin neoplasms and prevent the progress and spread of the disease through appropriate therapeutic actions using COX-2 enzyme inhibitors.

As the elderly groups (with many diseases) will account for a large part of the population pyramid in Iran in the near future and considering the variety of race and climat- 
ic conditions in different regions of the country, it is essential to perform extensive national studies to identify new techniques and laboratory applications to diagnose various diseases, particularly cancer, to ensure individual and social health and improve quality of life.

\section{Acknowledgments}

The present study was performed with the cooperation of the Department of Pathology, School of Medicine and Deputy of Health Management, Mazandaran University of Medical Sciences. The authors also thank the Pathology Laboratory technical personnel of Bu-Ali Sina hospital, especially Rahman Ghafari and Khatereh Moslemi for their technical collaborations.

This research was based on MD thesis of Dr. Yosef Moeini at the Department of Pathology, School of Medicine, Mazandaran University of Medical Sciences (registration number 345-2016). This study was approved by the Ethics Committee of Mazandaran University of Medical Science.

\section{Conflict of Interests}

The authors declare that they have no competing interests.

\section{References}

1. Dainichi T, Hanakawa S, Kabashima K. Classification of inflammatory skin diseases: a proposal based on the disorders of the three-layered defense systems, barrier, innate immunity and acquired immunity. J Dermatol Sci. 2014;76(2):81-9.

2. Siraj Ahmed S, Begum A, Shivaprasad P, Prabhu M. Incidence of Squamous Cell Carcinoma in Karnataka. Glob J Med Res. 2014;14(4).

3. Malekmohammadi M, Abdol Tehrani H, Aghdami N. Skin structure and wound healing phases. Journal of Dermatology and Cosmetic. 2011;2(4):229-44.

4. Robertson FM-L, Fitzgerald L. Skin cancer in the youth population of the United Kingdom. Journal of Cancer Policy. 2017;12:67-71.

5. Leiter U, Garbe C. Epidemiology of melanoma and nonmelanoma skin cancer - the role of sunlight. Sunlight, vitamin D and skin cancer: Springer; 2008. p. 89-103.

6. Queen L. Skin Cancer: Causes, Prevention, and Treatment. 2017.

7. Borutova R, Aragon YA, Nährer K, Berthiller F. Co-occurrence and statistical correlations between mycotoxins in feedstuffs collected in the Asia-Oceania in 2010. Anim Feed Sci Tech. 2012;178(3):190-7.

8. Sadler TW. Langman's medical embryology2015.

9. Kumar V, Abbas AK, Fausto N, Aster JC. Robbins and Cotran Pathologic Basis of Disease, Professional Edition E-Book: Elsevier Health Sciences; 2014.

10. Wolff K, Lowell A Goldsmith DMD, Paller A, Leffell DJ, Gilchrest BA, Katz SI. Fitzpatrick's Dermatology in General Medicine, Eighth Edition, 2 Volume set. Leffell DJ FD, editor: McGraw-Hill Education; 2012

11. Dubas LE, Ingraffea A. Nonmelanoma skin cancer. Facial Plast Surg Cl. $2013 \mathrm{Feb}$;21(1):43-53.

12. Cakir BO, Adamson P, Cingi C. Epidemiology and economic burden of nonmelanoma skin cancer. Facial Plast Surg Cl. 2012 Nov;20(4):419-22.

13. Quinn AG, Perkins W. Non-Melanoma Skin Cancer and Other Epidermal Skin Tumours. Rook's Textbook of Dermatology: WileyBlackwell; 2010. p. 1-48.

14. Afzali M, Mirzaei M, Saadati H, Mazloomi-Mahmood-Abadi SS. Epidemiology of skin cancer and changes in its trends in Iran. Feyz Journal of Kashan University of Medical Sciences. 2013;17(5):50111.

15. Mahmoodabad SS, Noorbala MT, Mohammadi M, Rahaei Z, Ehrampush MH. Knowledge, attitude, and performance of students toward skin cancer in Yazd, 2009. Int J Dermatol. 2011 Oct;50(10):1262-5.
16. Salehiniya H, Dashdebi SG, Rafiemanesh H, MohammadianHafshejani A, Enayatrad M. Time Trend Analysis of Cancer Incidence in Caspian Sea, 2004-2009: A Population-based Cancer Registries Study (northern Iran). Caspian J Intern Med. 2016;7(1):25.

17. Pakzad R, Soltani S, Salehiniya H. Epidemiology and trend in skin cancer mortality in Iran. J Res Med Sci. 2015;20(9):921-2.

18. Keyghobadi N, Rafiemanesh H, Mohammadian-Hafshejani A, Enayatrad M, Salehiniya H. Epidemiology and trend of cancers in the province of Kerman: southeast of Iran. Asian Pac J Cancer Prev. 2015;16(4):1409-13.

19. Kubus M, Andrzejewska E, Nejc D, Kobos J, Taran K. Evaluation of COX-2 protein expression in melanocytic nevi in children. Pol Merkur Lekarski. 2013;35(206):82-4.

20. Apalla Z, Lallas A, Sotiriou E, Lazaridou E, Ioannides D. Epidemiological trends in skin cancer. Dermatol Pract Concept. 2017;7(2):1.

21. Robati RM, Toossi P, Karimi M, Ayatollahi A, Esmaeli M. Screening for Skin Cancer: A Pilot Study in Tehran, Iran. Indian J Dermatol. 2014 Jan-Feb;59(1):105.

22. Eide MJ, Krajenta R, Johnson D, Long JJ, Jacobsen G, Asgari MM, et al. Identification of Patients With Nonmelanoma Skin Cancer Using Health Maintenance Organization Claims Data. Am J Epidemiol. 2010 Jan 1;171(1):123-8.

23. Michael AB, David MO. Squamous Cell Carcinoma of the Skin: Current Strategies for Treatment and Prevention. Curr Cancer Ther Rev. 2009;5(1):37-44.

24. Feller L, Khammissa R, Kramer B, Altini M, Lemmer J. Basal cell carcinoma, squamous cell carcinoma and melanoma of the head and face. Head Face Med. 2016;12(1):11.

25. Stewart BW, Wild CP. World Cancer Report 2014: International Agency for Research on Cancer; 2014.

26. WHO. (World Health Organization). "How common is skin cancer?" Retrieved 30 June 2014. Available from: http://www.who.int/uv/faq/skincancer/en/index1.html.

27. Robbins P. Skin Cancer Facts and Stats. (2016, June 8). Available from: http://www.skincancer.org/skin-cancer-information/skin-cancerfacts.

28. Enayatrad M, Mirzaei M, Salehiniya H, Reza M. Trends in Incidence of Common Cancers in Iran. Age. 2016;20:25.

29. Ferlay J, Soerjomataram I, Dikshit R, Eser S, Mathers C, Rebelo M, et al. Cancer incidence and mortality worldwide: sources, methods and major patterns in GLOBOCAN 2012. Int $\mathrm{J}$ Cancer. 2015;136(5):E359-86.

30. Maracy MR, Moradpour F, Hosseini SM, Tirani M. Cancer incidence and prevalence in isfahan: application of mortality data to estimates and projects for the period 2001-2015. Int J Prev Med. 2012;3(12):867-74

31. Mazloomy Mahmoodabad SS, Noorbala MT, Rahaee Z, Mohammadi M. Knowledge, attitude and performance study of secondary school teachers of Yazd city regarding skin cancer.

J Eur Acad Dermatol Venereol. 2010;24(4):424-8.

32. Millanta F, Asproni P, Canale A, Citi S, Poli A. COX-2, mPGES-1 and EP2 receptor immunohistochemical expression in canine and feline malignant mammary tumours. Vet Comp Oncol. 2016;14(3):270-80.

33. Bagir EK, Açikalin A, Izol V, Seydaoglu G, Erdogan S. Immunohistochemical Study of Cyclooxygenase-2 Expression in Prostate Carcinoma: It's Relation with Apoptosis and Angiogenesis. Bulletin of Urooncology. 2016;15(4):144

34. Morita Y, Morita N, Hata K, Nakanishi M, Kimoto N, Omata T, et al. Cyclooxygenase-2 expression is associated with vascular endothelial growth factor-c and lymph node metastasis in human oral tongue cancer. Oral Surg Oral Med Oral Pathol Oral Radiol. 2014;117(4):502-10.

35. Krishnamachary B, Stasinopoulos I, Kakkad S, Penet M-F, Jacob D, Wildes F, et al. Breast cancer cell cyclooxygenase-2 expression alters extracellular matrix structure and function and numbers of cancer associated fibroblasts. Oncotarget. 2017;8(11):17981.

36. Ghosh N, Chaki R, Mandal V, Mandal SC. COX-2 as a target for cancer chemotherapy. Pharmacol Rep. 2010;62(2):233-44.

37. Aeini F, Rezaei M, Ramezan Zadeh R, Pooladi A, Nik'khu B. Study of Cyclooxygenase-2 Expression in Sprague Dawley Rat Gastric Cancer Induced by H. Pylori. Qom Univ Med Sci J. 2011;4(4):3-9.

38. Jiao J, Ishikawa T-o, Dumlao DS, Norris PC, Magyar CE, Mikulec $\mathrm{C}$, et al. Targeted Deletion and Lipidomic Analysis Identify Epithelial 
Cell COX-2 as a Major Driver of Chemically-induced Skin Cancer. Mol Cancer Res. 2014;12(11):1677-88.

39. Becker MR, Siegelin MD, Rompel R, Enk AH, Gaiser T. COX-2 expression in malignant melanoma: a novel prognostic marker? Melanoma Res. 2009;19(1):8-16.

40. Zelenay S, van der Veen AG, Böttcher JP, Snelgrove KJ, Rogers N, Acton SE, et al. Cyclooxygenase-dependent tumor growth through evasion of immunity. Cell. 2015;162(6):1257-70.

41. Pires I, Garcia A, Prada J, Queiroga F. COX-1 and COX-2 expression in canine cutaneous, oral and ocular melanocytic tumours.

J Comp Pathol. 2010;143(2):142-9.

42. Korgavkar K, Xiong M, Weinstock MA. Compact fluorescent lamps and risk of skin cancer. J Cutan Med Surg. 2013;17(5):308-12.

43. Andreassi L. UV exposure as a risk factor for skin cancer. Expert Review of Dermatology. 2011;6(5):445-54.

44. Kuzbicki L, Lange D, Straczynska-Niemiec A, Chwirot BW. The value of cyclooxygenase-2 expression in differentiating between early melanomas and histopathologically difficult types of benign human skin lesions. Melanoma Res. 2012;22(1):70-6.

45. Souza do Nascimento J, Carlos R, Delgado-Azañero W, Mosqueda Taylor A, Almeida OP, Romañach MJ, et al. Immunohistochemical expression of cyclooxygenase-2 (COX-2) in oral nevi and melanoma. J Oral Pathol Med. 2016;45(6):440-3.

46. Razi S, Rafiemanesh H, Ghoncheh M, Khani Y, Salehiniya H. Changing trends of types of skin cancer in Iran. Asian Pac J Cancer Prev. 2015;16(12):4955-8. 